\title{
Prevention and Early Detection of Acute Kidney Injury in Intensive Care Unit: A Systematic Review
}

\author{
Thandar Soe Sumaiyah Jamaludin ${ }^{1}$, Mohd Said Nurumal ${ }^{1 *}$, Muhammad Kamil Che Hasan ${ }^{1}$, Siti \\ Hajar Shah Rizuan², Nur Fadhlin Farhanah Mohamed Faizal ${ }^{3}$ \\ ${ }^{1}$ Kulliyyah of Nursing, International Islamic University Malaysia, Pahang, Malaysia. \\ 2Subang Jaya Medical Centre, Selangor, Malaysia. \\ ${ }^{3}$ National Heart Institute, Kuala Lumpur, Malaysia.
}

\begin{abstract}
Acute Kidney Injury (AKI) is often associated with Intensive Care Unit (ICU) admission and results in a higher mortality rate around the world. The AKI cases have been rising for the past decade alongside the mortality rate. Literature has shown that early detection helps in overcoming the problem. This systematic review aims to identify the identify the relevant literature and to summarize the prevention and early detection of AKI in the ICU setting to reduce the morbidity and mortality rate of patients. A total of 20 articles identified in this review. This systematic review also is in line with the Preferred Reporting Items for Systematic Reviews and Meta-Analyses (PRISMA) guidelines. Relevant studies were identified based on the inclusion criteria with language filters but were limited to articles published from the year 2010 to 2020. Two reviewers independently conducted the quality assessment, data extraction, and analysis on all included studies. The result indicates there are several biomarkers which are helpful in detecting AKI such as Neutrophil Gelatinase-Associated Lipocalin (NGAL), Interleukin-18 (IL-18), Tissue Inhibitor of Metalloproteinase 2 (TIMP-2), Insulin-Like Growth Factor Binding Protein 7 (IGFBP-7), Protein C (PC), and soluble thrombomodulin (sTM). This review also addressed nursing responsibilities for early detection and prevention of AKI. Thus, severe cases of AKI and mortality cases associated with AKI can be minimized with better predictive and proper management.
\end{abstract}

Keywords: Acute kidney injury; Intensive care unit; Prevention; Early detection

\section{*Corresponding author}

Assoc. Prof. Dr. Mohd Said Nurumal

Department of Critical Care Nursing,

Kulliyyah of Nursing,

International Islamic University Malaysia,

Jalan Sultan Ahmad Shah, Bandar Indera Mahkota,

25200 Kuantan, Pahang, Malaysia.

Email: mohdsaid@iium.edu.my

\section{Article History:}

Submitted: 16 December 2021

Accepted: 22 January 2022

Published: 31 January 2022

ISSN: 2600-898X 


\section{INTRODUCTION}

Acute Kidney Injury (AKI) is a common complication of deterioration of kidney function commonly seen among critically ill patients in an intensive care unit (ICU). AKI is inevitably linked with complex pathophysiology due to varied underlying diseases in critically ill patients. Moreover, AKI is a progressive disease and there are three stages of AKI; namely, stage 1, stage 2 and stage 3 can be seen in the ICU based on the severity of their conditions (1). A study reported that stage 3AKI had lower survival compared with other AKI stages and required the importance of early detection of AKI in ICU (2).

AKI also has shown a significant rise from 2005 to 2014 both in males and females from $6.1 \%$ to $13.2 \%$ and $5.0 \%$ to $11.5 \%$ respectively, thus in need of attention for AKI events as well as their determinants (3). A higher mortality rate among AKI patients in ICU is a major health problem. It is reported that $50 \%$ of the death caused by AKI had received suboptimal care and $14 \%$ of the death is found avoidable (4). Besides, AKI is frequently associated with ICU admission, intubation and re-intubation, and re-admission of ICU compared to other medical problems (5-7). Studies have also shown that patients with AKI have 3 times higher mortality compared to those who don't have ( $2 \& 8)$. Therefore, to reduce the mortality rate of AKI patients, early detection, prevention and early treatment are crucial in managing AKI (3).

A study also found that the knowledge gap among the health care providers attributed to the morbidity and mortality rates of patients with AKI especially in the ICU (9). Therefore, it is important for nurses especially for ICU nurses to have a better understanding of prevention and early detection of AKI in ICU to promote the quality of life of patients (9-11). The delay in the detection of AKI is highly associated with not having a clear reference in defining the AKI (4). Furthermore, a slight increase in serum creatinine level can lead to an increase in the risk of death of the patient (5). AKI is a progressive disease; it is essential for healthcare providers especially nurses need to prevent and detect the disease at an early stage to increase the chance of a good prognosis of the disease as well as their quality of life (8-11). However, not having a clear reference for AKI might hinder the prevention of the disease. Confusion on determining the definition of AKI among the healthcare providers has led to the development of a few systems to help the healthcare practitioners in deciding the best treatment and care needed by their patients with AKI (8).

Even though AKI is highly associated with poor prognosis and a high mortality rate, it is preventable and treatable. Interventions to improve the diagnosis of AKI need to be done, considering the high number of often missed diagnosis rates of AKI (12). Therefore, to tackle this issue, awareness and knowledge on the prevention and early detection of AKI need to be discussed further to reduce the incidence of patients with AKI in ICU settings. Thus, this paper focused on the prevention and early detection of AKI in ICU.

\section{METHODS}

The aimed of conducting this systematic review was to identify the relevant literature and to summarize the prevention and early detection of AKI in the ICU setting to reduce the morbidity and mortality rate of patients. Furthermore, we have included the studies examining related to predictive biomarkers and preventive measures of AKI, together with reviewing the best methods for both of them in this review.

\section{Data sources and literature search strategies}

The searching of literature was performed by using electronic data bases such as ProQuest, ScienceDirect and Scopus. The following searched keywords for this review were used in separately and combination with each others. Those were intensive care unit, critical ill patients, critically ill patients, critical unit care, acute kidney injury, acute renal failure, prevention, early detection. The literature search limit include human, English language, adult aged 18 years and above, publication date 2010 to 2021 but no limit were imposed on characteristics of studied participants, design of the study, intervention of the study and methodology of the study that used by the previous researchers. The author and the team also did hand search for references list of all allocated studies for further related studies. 


\section{Selection Criteria}

All studies were included if they met the inclusion criteria such as their recruited participants with AKI, participants with high risk for having AKI, participants who are critically ill with high risk for having AKI and undergoing treatment in ICU, and the studies have carried out for predictive biomarkers and preventive measures of AKI. The following elements were excluded in this review for instance the study report on identified editorials, reported on studies of end stage renal failure and other groups such as participant on regular haemodialysis or continuous renal replacement therapy receiving in ICU. Furthermore, studies with no detail's information were also excluded in this review. The review of clinical question for this study was defined using PICO framework; population $(\mathrm{P}), \quad$ intervention $(\mathrm{I})$, control/comparison (C), and outcome of interest $(\mathrm{O})$ as shown in the Table I (13).

Table I: PICO framework for article review

\begin{tabular}{|l|l|}
\hline PICO element & Inclusion criteria for review \\
\hline Population & $\begin{array}{l}\text { Critically ill patients } \\
\text { Adult patients with AKI or } \\
\text { having high risk of AKI }\end{array}$ \\
\hline Intervention & $\begin{array}{l}\text { AKI; preventive measures } \\
\text { AKI; early detection }\end{array}$ \\
\hline Control/ & $\begin{array}{l}\text { Using urine and plasma } \\
\text { biomarker for early detection of } \\
\text { AKI } \\
\text { Monitoring of urine output and } \\
\text { prevention of AKI } \\
\text { Monitoring of haemodynamic } \\
\text { status and early detection/ } \\
\text { prevention of AKI }\end{array}$ \\
\hline Outcome & $\begin{array}{l}\text { AKI mortality rate/morbidity } \\
\text { rate } \\
\text { Management of AKI } \\
\text { Prevention and early detection }\end{array}$ \\
\hline
\end{tabular}

Data Extraction and search outcomes

The available data were extracted from studies meeting with eligibility criteria of patient with AKI and patients with high risk for having AKI. The main outcome measures for this review were prevention and early detection of AKI in ICU while the secondary outcome were predictive biomarkers and preventive measures of AKI. All data were extracted for characteristics of subject included, condition of recruitment, measures used to assess AKI prevention and early detection in ICU, main method of analysis and the strength association predictive biomarkers, haemodynamic status monitoring to detect $\mathrm{AKI}$, preventive measures of AKI in ICU. Four hundred and sixty-seven articles were identified from the chosen electronic data bases and non from other sources. Two hundred and eighty-one articles were excluded after reading as they were duplicate articles. After screening all articles from the rest, finally twenty articles have extracted and analysed for this review. Figure I illustrate the review process based on the PRISMA Flow Diagram.

Quality appraisal and assessment of included studies

In this review, the quality critical appraisal and assessment of the included studies have been carried out with the guideline from the Joanna Briggs Institute Critical Appraisal tools. The Joanna Briggs Institute Critical Appraisal tool was used in this review since this tool was succinct and effectively cover the areas needed for critical appraisal of evidence. The assessment all articles included in this review were checked with following criteria;

1. Inclusion criteria

2. Study subjects and settings

3. Valid and reliable exposure measure

4. Objectives and standard criteria used for measurement of the condition

5. Confounding factors

6. Strategies to addressed confounding factors

7. Valid and reliable measurement used for outcomes

8. Appropriate statistical analysis

According to the above criteria, the quality of each study was determined by two authors and any uncertain issues were resolved by coauthors. The assessment of included studies are graded as 'yes', 'unclear' and 'no' represents poor quality. The studies are rated according to the number of 'yes' defined as higher quality, 'unclear' as medium quality and 'no' indicating poor quality. Moreover, during the assessment of included studies, if the study was graded as 'yes' more than 5 accounted as strong, 'yes' 3 until 5 regarded as moderate and 'yes' less than 3 referred as poor. If a study has 'no' more than 
Figure I: Review process based on PRISMA flow diagram
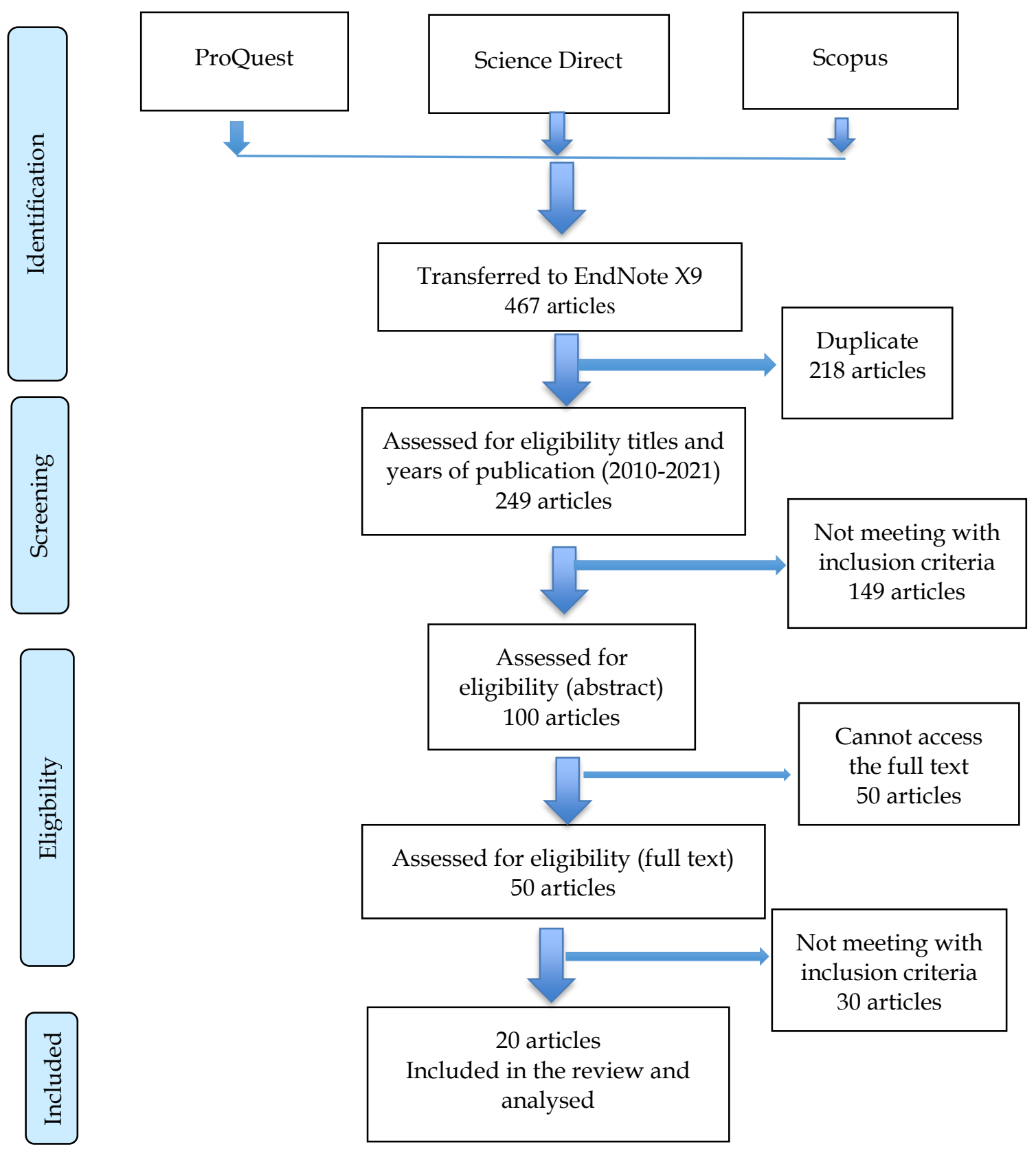
3 or more than 4 for 'unclear', the articles deemed ineligible to be used. Any disputes in judgments when choosing the articles are resolved using discussion among the authors. Among the 20 articles that selected in this review, 8 studies have strong agreement between authors and the other 12 studies have moderate agreement between authors. Most of the included studies do not mention confounding factors and ways to deal with it.

\section{Data synthesis}

In the review process, searched studies were synthesized with the following subheading: author, date of publication, study design, sample size with sampling method and respond rate, data collection instrument, and key findings. The data on predictive biomarkers and preventive measures of AKI were subsequently reviewed. Findings of this review are further described in the following session.

\section{RESULTS}

\section{Characteristics of the included studies}

Twenty articles which included in this review were generally assessed based on several aspects which address the aimed of this systematic review. Among the 20 articles, only 3 articles were done in Malaysia. The sample size for included studies ranged from 80 to 4049. Most of the study designs used in the included studies were retrospective cohort and prospective cohort studies. The following section describe further on the findings of this review.

\section{Risk of having acute kidney injury (AKI)}

AKI is a major health problem which affect the survival of the patients and associated with ICU admission (5 \& 11). The AKI highest detection rate accounted for $22.46 \%$ in ICU, followed by medical and surgical departments with $1.96 \%$ and $0.86 \%$ respectively (11). Studies2 \& also found that the main risk factors of AKI development were patients who are having sepsis and history of developed shock (2\&14). According to national institute for health and care excellence, patients who have high risk of developing AKI are those who are more than 65 years old, having heart failure, liver disease, chronic kidney disease specifically with eGFR less than 60 , past history of AKI, diabetes, neurological impairment specifically when the patient rely on a carer which may mean reduced access to fluid, hypovolaemia, oliguria defined as urine output less than $0.5 \mathrm{ml} / \mathrm{kg} /$ hour, haematological malignancy, symptoms of urological obstruction, sepsis, history of the usage of iodinated contrast agents within the past week, on medication with nephrotoxic potential and deteriorating early warning score (EWS) (15).

Early detection of acute kidney injury (AKI) in ICU Deterioration of renal in AKI obstructs glomerular filtration, thus acts as biomarker in order to detect the AKI. The importance of each AKI biomarker for investigation purposes need to be understood to discern the physiology of AKI. Thus, the following predictive biomarkers are commonly used to detect the AKI.

\section{Urine Output (UO) Monitoring}

Kidney disease improving global outcomes (KDIGO) is the best tool to predict AKI with great classification scoring (16). One of the included criteria for KDIGO is decreasing urine output, yet there is no agreement in the method of measuring urine output (17). However, a study conducted to compare two urine collection methods, which are; urine output in each consecutive hour (UOcons) and mean hourly urine output (UOmean) (17). Their result showed that the UOcons has little impact on the incidence of stage 2 and stage 3 in AKI based on KDIGO criteria and vice versa for UOmean (17). They further stated that he urine output measured independently from $\mathrm{SCr}$ is rather less effective in diagnosing AKI (17).

A study has reported about the intensive monitoring of urine output (UO) of ICU patients that the crude rates of AKI (stage 2-3) by 7 days after patient's ICU admission and patients with intensive monitoring of UO was higher (OR, 1.22; 95\% CI, 1.11-1.35; $\mathrm{P}<.001$ ) compared to patients with less intensive UO monitoring (18). Intensive UO monitoring and less intensive UO monitoring in this article was defined as hourly monitoring and no gaps of more than 3hours for the initial 48 hours after ICU admission and daily monitoring respectively (18). Moreover, patients with AKI who undergo intensive UO recording was strongly associated with improved survival to 30 days (OR, 1.22; 95\% CI, 1.11-1.35; $\mathrm{P}<.001$ ) and low less proportion of $\geq 10 \%$ fluid overload 
( $2.97 \%$ vs $7.13 \%$; $\mathrm{P}<.001)$ compared to patients with less intensive UO recording (18).

Serum Creatinine (SCr)

A study also described that the $\mathrm{UO}$ and serum creatinine (SCr) is one of the best biomarkers used in AKI detection (15). Kidneys are responsible for filtering out creatinine, a waste product produced by muscles, out of the body, thus, an elevated level of $\mathrm{SCr}$ indicates a reduced kidney function. Normal creatinine level is $60-120$ micromol/L which is widely ranged as the amount of creatinine secreted depends on the patient's muscle bulk (15). Thus, it is recommended that regular $\mathrm{SCr}$ monitoring is a must for patients with a high risk of AKI development (15).

\section{Neutrophil Gelatinase-Associated Lipocalin (NGAL) and Interleukin-18 (IL-18)}

Aside from measuring UO and SCr, Neutrophil Gelatinase-Associated Lipocalin (NGAL) secreted in response to tubular injury is the most well-known to be used as an AKI detector (19). The NGAL can be measured in urine taken from a catheter or plasma taken from blood in venous or arterial lines (19). Besides, the NGAL in urine and found out it was an accurate predictive biomarker for AKI in cardiac surgery, coronary angiography patients and cirrhotic patients (19\&20). A study also found that the NGAL taken from urine from patients undergoing liver transplants acts as a better AKI independent predictor compared to NGAL from blood plasma (21).

Moreover, the NGAL from urine also can predict the patients' needs for renal replacement therapy and much more optimal in diagnosing the AKI in patients with sepsis than in non-infectious systemic inflammatory response syndrome (SIRS) (22). Another study found that cardiac postoperative patients, urine NGAL is not as effective in their cohort compared to plasma NGAL and urine Interleukin-18 (IL-18) (23). However, a study found that patients undergoing cardiac surgery using cardiopulmonary bypass also showed an equivalent result with plasma NGAL outperformed urine NGAL in defining AKI (24). Researchers have mentioned that both early postoperative levels of urine IL-18 and plasma NGAL are highly associated with AKI, mortality, hospital length of stay after cardiac surgery $(23 \& 24)$. A postoperative level of urine
IL-18>60 $\mathrm{pg} / \mathrm{ml}$ denoted more than 6-fold risk of AKI compared with patients in the lowest quintile, whereas a postoperative level of plasma NGAL $>293 \mathrm{ng} / \mathrm{ml}$ denoted a 5 -fold risk of AKI (23).

Tissue Inhibitor of Metalloproteinase 2 (TIMP-2) And Insulin-Like Growth Factor Binding Protein 7 (IGFBP-7)

Tissue inhibitor of metalloproteinase 2 (TIMP2) and insulin-like growth factor-binding protein 7 (IGFBP-7) in urine specimens or NephroCheck Test are also known as 'renal alarm'. TIMP-2 and IGBF-7 may elevate when there is inflammation, oxidative stress and exposure to drugs and toxins that can cause AKI, signalling the kidney's attempt in protecting itself from harm (25). They reported on the prediction the development of moderate-to-severe AKI in the next 12 to 24 hours using TIMP-2 and IGFBP-7 in surgical ICU that out of 375 patients included in their final analysis, $35(9 \%)$ of them have developed moderate-severe AKI within 12 hours, proving that these biomarkers performed well in detecting pre-AKI (25). However, as study found that the median level of urine TIMP-2 and IGFBP-7 was significantly higher, it was inadequate to independently predict AKI (20). But the researcher was unable to state the exact reason for the outcome and assumed the possible mechanism is the production of damaged hepatocytes in chronic liver disease. Protein C (PC) and soluble thrombomodulin (sTM) Endothelial dysfunction is an early episode for AKI and Protein C (PC) and soluble thrombomodulin (sTM) are markers for endothelial dysfunction (26). A study investigated the relationship of PC and STM in AKI patients defined by AKIN creatinine (AKI Scr) urine output criteria (AKI UO) and the result obtained showed a rise in sTM levels 24 hours before in AKI Scr then proceeded to slightly declining afterwards (26). Elevated sTM is a strong independent predictive element for AKI (27). Regarding PC, there were no significant changes before and after AKI diagnosis. PC levels are mostly used to assess patients with sepsis and septic shock over the last decades, however, the PC levels were not different in patients with and without AKI (26). They also tested the relationship between PC and sTM with mortality as well as non-renal recovery and PC levels were independently associated with the event. Regardless of those 
significant changes in AKI Scr patients, there were no changes in AKI patients with AKI UO alone. This concludes that PC and sTM levels will help determine the pathway involved in AKI since $\mathrm{Scr}$ alteration is related to the glomerular filtration rate $(26 \& 27)$.

\section{Prevention of AKI in ICU}

Early detection for AKI is important as early detection means planning care for AKI sooner thus stopping the progression of the disease, promising a good prognosis for the patients. Nurses need to play their role in the early detection and prevention of AKI especially for patients who are having high risk of developing AKI. Therefore, the following preventive measures are commonly used to prevent the occurrence of AKI.

\section{Urinary pattern and symptoms monitoring}

Dehydration is the most common cause of community-acquired AKI (15). Thus, monitoring patients' changes in urine output are important in the early detection of AKI, especially in ICU. In order to predict the possibility of $\mathrm{AKI}$, identifying the volume of urine passed, investigating the differences and changes in the fluid intake and output and also the differences in the patient's weight (28). A previous study also stated that the patient's fluid volume status needs to be carefully assessed for both fluid depletion and fluid overload (29). Nevertheless, nurses should take note of haematuria, dysuria or pyuria and urgency, dribbling and incontinence, and changes in creatine level since a slight rising creatinine with Fractional excretion of sodium (FENa) in urine composition more than 2.0\% highly suggests renal AKI (28 \& 30).

\section{Haemodynamic parameters}

Monitoring of haemodynamic parameters are important for critically ill patients in ICU. A study found that hypotension systolic blood pressure (SBP) less than $110 \mathrm{mmHg}$ and mean arterial pressure (MAP) of less than $65 \mathrm{mmHg}$ need immediate assessment and IV fluid challenges to avoid any possible progression of AKI (29). History of dizziness and orthostatic volatility are related closely to intravascular reduction and the presence of oedema, weight gain and periorbital swelling might indicate fluid retention (28). Thus, it is important to monitor if there is any fluid loss and investigate the causes if it has resulted from haemorrhage, polyuria, vomiting and diarrhea as it can result in excessive insensitive losses (28 \& 29). Moreover, using Early Warning Score (EWS) which composite score of six bedside vital parameters: pulse rate, blood pressure (BP), oxygen saturation (SpO2), body temperature, respiratory rate, and mental state, should be implemented in every clinical setting as it alerts the nurses if the patient's health is deteriorating or already at the risk of deteriorating (15). Furthermore, the role of nurses to be alert if their patient's blood pressure is reduced, as the patient's UO will likely be reduced below $0.5 \mathrm{ml} / \mathrm{kg} / \mathrm{h}$, indicating one of the signs of preAKI (28 \& 29).

\section{Iodinated Contrast Media Administration}

Iodinated contrast media used during clinical diagnostic radiology can cause vasoconstriction in the renal artery thus altering glomerular haemodynamic due to the elevation of plasma oncotic pressure thus leading to contrast-induced AKI (15). This is harmful to patients with a known risk of AKI. It is recommended that volume expansion and isotonic sodium carbonate or $0.9 \%$ sodium chloride should be given to these patients before proceeding with the clinical diagnostic radiology (15).

\section{Medication Issue}

Nurses need to be alert on medications that can harm their patient's kidneys as few medications are well known for impairing kidney function. Nurses should assess if their patient took any herbal products, over-thecounter medications or any health supplements as some herbal products for example mutong and fangchi contain aristolochic acid which could result in Acute Interstitial Nephritis (AIN), which is one of the major causes of AKI (15). Nephrotoxic drugs are one of the important assessments in patients with potential kidney impairments. The drugs that are highly associated with AIN are antibiotics such as methicillin, cephalosporins, sulphonamides, rifampicin, erythromycin, and ciprofloxacin, diuretics including furosemide, thiazides and chlorthalidone, non-steroidal anti-inflammatory drugs (NSAIDs), anticonvulsant drugs for including phenytoin and carbamazepine and allopurinol (15). Nephrotoxic drugs might accumulate inpatient's renal cortex and if it is highly concentrated, it will cause vasoconstriction, 
leading to acute tubular necrosis. They have also recommended that Angiotensinconverting enzyme (ACE) inhibitors and angiotensin-receptor II blockers (ARBs) should not be administered to patients with a high risk of AKI as these medications will promote and exacerbate renal impairment because of the reduction of perfusion pressure resulting by the drugs (15). A study also suggested that a temporary cessation for ACE and ARB drugs for dehydrated and hypotension, systolic blood pressure less than $110 \mathrm{mmHg}$ patients (29). Moreover, the medications such as tricyclic antidepressant also may precipitate or lead to urinary retention. This assessment is important in the initial diagnosis and planning the further management of AKI (15, 28 \& 29).

\section{DISCUSSION}

Kidney is an organ responsible for maintaining fluid balance in our body by filtering the blood and excreting waste product. An injury to the kidney will result in lesser output excreted from the body which will results in AKI and its complication. Thus, by knowing how to detect early on their symptoms and how to prevent them is essential responsibilities for every healthcare provider. Studies have shown that lack of understanding of kidney function among the attending healthcare providers leads to the neglection of early symptoms of AKI thus delaying the detection of AKI, denying its further management and worsening its prognosis $(5,9 \& 12)$.

\section{Risk of having acute kidney injury (AKI)}

Based on the findings of this review, the major risk factors contributing to AKI in ICU setting are sepsis and shock, followed by older age, history for having chronic diseases including heart failure, liver disease, diabetes and chronic kidney disease, history of the usage of iodinated contrast agents within the past week, on medication with nephrotoxic potential and deteriorating early warning score (EWS) (1621). In addition to previous groups, nurses should pay more attention to patients with a history of usage of iodinated contrast agents, on medication with nephrotoxic potential and deteriorating early warning score (EWS) as well (18-20). Thus, nurses, especially those who work in ICU need to be equipped with the knowledge of risk factors of AKI to help them stay alert with any pre AKI symptoms shown by the patient.

Early detection of acute kidney injury (AKI) in ICU Given that early detection of AKI is extremely important, there are several studies researching AKI biomarkers have found in this review. For instance, RIFLE, AKIN and KDIGO are widely used in defining AKI stages by measuring $\mathrm{SCr}$ and urine output. A study was done to determine the specific ways in measuring urine output, either by measuring the urine output in each consecutive hour or taking the mean hourly for urine output and the latter resulted in overdiagnosis for AKI stage 2 and 3 (18). This finding suggests that more AKI patients who went through intensive UO monitoring are managed with a more conservative volume resuscitation strategy if compared to patients with less intensive UO monitoring (17). They further suggested that measuring urine output hourly which was considered as intensive monitoring as it is strongly associated with improved survival among AKI patients. On the other hand, elevated SCr highly represents kidney failure and it is importance of $\mathrm{SCr}$ by mentioning the urine output measured independently from $\mathrm{SCr}$ is rather less effective in diagnosing AKI (17 \& 18).

Aside from two main biomarkers, which are $\mathrm{SCr}$ and urine output, several studies are done on other biomarkers to figure out the best AKI predictor. One of them is NGAL which can be collected either by blood plasma or urine. There are several studies that believed that NGAL collected from urine is a better independent predictor than blood plasma based on his study (19-21). However, a study suggested to choose to go for the vice versa and reported NGAL taken from blood plasma is more sensitive to renal tubular injury (23). The author then continued by mentioning the effectiveness of IL-18 in defining AKI alongside plasma NGAL. Despite the arguments between both types of NGAL, all studies are agreed on the effectiveness of NGAL in determining AKI. On the contrary, a study agreed to differ by claiming STM is able to categorise AKI SCr defined by AKIN classification better than NGAL, yet unable to give significant results for AKI UO (26). Therefore, the author then concluded STM is exceptionally helpful in determining the pathway involved in AKI patients. Other biomarkers being researched 
are TIMP-2 and IGFBP and both performed remarkably well in detecting pre-AKI (25). However, a study obtained a different result in cirrhotic patients as both TIMP-2 and IGFBP-7 were inadequate to independently predict $\mathrm{AKI}$ (20).

Studies have mentioned that both early postoperative levels of urine IL-18 and plasma NGAL are highly associated with AKI, mortality, hospital length of stay after cardiac surgery (23\&24). A postoperative level of urine IL-18>60 pg/ml denoted more than 6-fold risk of AKI compared with patients in the lowest quintile, whereas a postoperative level of plasma NGAL > $293 \mathrm{ng} / \mathrm{ml}$ denoted a 5-fold risk of AKI (23). This result suggests that the utilisation of this biomarkers which are well known with its sensitivity to renal tubular injury (25 \& 31) will change the prognosis of postoperative patients, specifically cardiac surgery patients who are having high risk for AKI.

\section{Prevention of AKI in ICU}

It is wise to stop something from happening instead of dealing with it later, as prevention is always better than cure. ICU patients are known for having a high risk of AKI thus lengthening their ICU stay and worsening their condition. According to the findings of this review, few issues on preventive measures have been addressed. Mainly, the issue highlighted from the articles is the importance of thorough nursing assessment. Nurses need to be alert of their patient's condition, especially critically ill patients in ICU to ensure their better prognosis also avoid further unnecessary casualties.

Since changes in the composition of urine can indicate a certain illness, it is important for nurses to observe the patient's urinary pattern. The most important preventive measure for patients that show the risk of AKI that can be done is close monitoring of the patient's urinary pattern and symptoms. Nurses should take account of the changes in their patient's urine output and any records of changes of the composition of the urine, especially any signs of haematuria, dysuria, pyuria and urgency, dribbling and incontinence. This is followed by close monitoring of the patient's haemodynamic pattern. Any obvious changes of blood pressure that need to be noted by the nurse's in charge as slight changes in critically ill patients are important. Early Warning Score (EWS) is also handy for nurses to observe any abnormality of the patients in their care. Nurses need to be alert and understand how these scores work in order to be able to notice their patient's vital signs abnormalities, especially when patient's blood pressure is reduced as it is most likely that their patient's UO is reduced below $0.5 \mathrm{ml} / \mathrm{kg} / \mathrm{hr}$ which shows the high possibility of a patient is at risk of AKI.

Nurses need to alert the physicians involved if their critically ill patients need to undergo any clinical diagnostic radiology where iodinated contrast media is involved as it may exacerbate the progress of AKI. For medication issues, nurses should assess the patient's medication if they have taken any herbal product or supplements that may harm their kidney in detecting patients with a high risk of AKI. Nurses need to alert the physicians to avoid administering nephrotoxic drugs that may harm the kidney and worsen the prognosis of patients with a high risk of AKI. Moreover, nurses need to have upgraded in their knowledge by doing evidence-based research, subscribing the latest research articles from nursing and medical journals, using internet for their academic improvement in order to prevent AKI happening in their patients (32 34). These skills for nurses are important it was due to an increase in the number of patients with a wide range of problems and symptom in clinical setting, nurses are also required to equip knowledge on the treatment of acute and chronic diseases and preventing further complications such as diabetes and wound care (35\& 36). In some cases, patient with chronic diseases such as diabetes has higher chance of developing AKI (20).

Nurses should assess any sign and symptoms of infection as infection is highly associated with AKI (28). This is supported by previous study where they emphasised the primary role of nurses to be alert and suspect AKI probability in patients who developed signs of sepsis (15). From this evidence, it is clear that nurses need to be well equipped with signs and symptoms of sepsis to fulfill their role in detecting early signs of pre-AKI and AKI. Nurses should also assess if their patients have any history of systemic disease symptoms. This is important in detecting patients with a high 
risk of AKI, thus management to prevent AKI can be planned beforehand. Assessing these symptoms can help the nurses to identify patients with high probability of AKI or already having pre-AKI symptoms, thus future management to prevent it from happening can be arranged.

\section{Limitation}

One of the limitations is that fewer Malaysianbased articles on predictive biomarkers and preventive measures of AKI can be found or yet to be published. This is considered a limitation since gene and cultural factors are slightly different in every ethnicity and should not be generalised to western standards. Another limitation that can be found from this literature review is there is a lack of published articles on nursing management for pre-AKI patients in the local setting, leading to the increase of delayed detection of AKI. There are also no articles on the risk of pre-AKI patients that can be found in the local context especially in ICU settings. However, the research done worldwide could still be used as references.

\section{Recommendation}

After completing of this literature, the authors would like to suggest to have future further studies to continue the research and address the particular matter. Aside from that, the total articles reviewed might be inadequate due to limited databases used, nevertheless, all articles are matched with inclusion and exclusion criteria. Larger studies with more amount of articles should be carried out to have a better evaluation on this issue. It is also recommended that studies on predictive biomarkers and preventive measures of AKI be conducted in a local context to overcome the high mortality due to AKI in Malaysia. Furthermore, it is also suggested that more studies should be conducted on nursing management for preAKI patients in a local setting. Future studies for patients with the risk of pre-AKI especially in ICU settings should also be conducted in Malaysia.

\section{CONCLUSION}

AKI incidence is a concern in a healthcare system, yet there are still hopes for improvement. The previous studies covered serum creatinine and urine output in defining AKI, though there are more biomarkers that could help in detecting AKI in the early stages. Many articles can be found especially on NGAL either plasma or urine showing the effectiveness of NGAL in distinguishing AKI. Despite that, sTM is claimed to have better results than NGAL and there are biomarkers such as TIMP-2 and IGFBP-7 which also show the excellent outcomes. Aside from early detection, preventive measures are also essential in managing AKI. Monitoring urinary patterns, haemodynamic changes, EWS, signs of infection and systemic disease symptoms are a part of nursing responsibilities in dealing with AKI patients. Apart from that, nurses should be alert with patients' medication and if any critically ill patients are in need of iodinated contrast media administration as it may induce AKI especially in critical care settings.

We are expecting that this review findings might help healthcare providers in the future aside from aiding the ICU in planning better management for patients with AKI in order to reduce the length of stay by AKI patients and to decrease the mortality incidence resulting from AKI. Apart from that, this study could also be contributed to other departments to refine their management for AKI patients, especially Emergency Departments, which requires an accurate diagnosis and fast treatment to be applied.

\section{CONFLICT OF INTEREST}

The authors acknowledge that the publishing of this paper does not have any conflict of interest.

\section{REFERENCES}

1. Acute Kidney Injury (AKI) - KDIGO. Retrieved March 19, 2020, from https://kdigo.org/guidelines/acutekidney-injury/. 2011.

2. Md Ralib A \& Mat Nor MB. Acute kidney injury in a Malaysian intensive care unit: Assessment of incidence, risk factors, and outcome. Journal of Critical Care. 2015; 30(3), 636-642. https://doi.org/10.1016/j.jcrc.2015.01.018

3. Dramatic Rise in AKI Incidence Rates Reported. Renal \& Urology News. 2018;17(5), 2-3. Retrieved from 
http://210.48.222.80/proxy.pac/docview $/ 2131138505$

4. Teo SH, Lee KG, Koniman R, Tng ARK, Liew ZH, Naing TT, Kaushik M. A prospective study of clinical characteristics and outcomes of acute kidney injury in a tertiary care Centre. BMC Nephrology. 2019; 20(1), 1-8. https:// doi.org/10.1186/s12882-019-1466z

5. Tang X, Chen D, Yu S, Yang L \& Mei C. Acute kidney injury burden in different clinical units: Data from nationwide survey in China. PLoS ONE. 2017; 12(2), 1-13. https://doi.org/10.1371/journal.pone.017 1202

6. Jamaludin TSS, Suhaimi NS, Mohd Noor H \& Nurumal MS. Intensive Care Unit (ICU) Readmission: A Mixed-Method Systematic Review. International Journal of Care Scholars 2021; 4(2).

7. Jamaludin TSS, Mohd Said Nurumal, Nur Syila Syahida Syaziman, Syuhada Suhaimi \& Muhammad Kamil Che Hasan. ReIntubation Among Critical Care Patients: A Scoping Review. International Journal of Care Scholars 2021; 4 (Supplementary 1).

8. Hoste EAJ, Bagshaw SM, Bellomo R, Cely $\mathrm{CM}$, Colman R, Cruz DN, Kellum JA. Epidemiology of acute kidney injury in critically ill patients: the multinational AKIEPI study. Intensive Care Medicine. 2015; 41(8), 1411-1423. https://doi.org/10.1007/s00134-015-39347

9. Adejumo OA, Akinbodewa AA, Alli OE, Pirisola OB \& Abolarin OS. Brief Communication Knowledge of Acute Kidney Injury among Nurses in Two Government Hospitals in Ondo City, Southwest Nigeria. In Saudi J Kidney Dis Transpl. 2017; (28). Retrieved from http://www.sjkdt.org

10. Jamaludin TSS \& Chan CM. Influence of Perceive Social Support or Pre-Operative Support Program on Quality of Life in Patients Awaiting Coronary Artery Bypass Grafting (CABG) Surgery: Mixed Method Systematic Review. International Journal of Care Scholars. 2019; 2 (1).

11. Singbartl K \& Kellum JA. AKI in the ICU: Definition, epidemiology, risk stratification, and outcomes. Kidney International. 2012; 81(9), 819-825. https:// doi.org/10.1038/ki.2011339

12. Cheng X, Wu B, Liu Y, Mao H \& Xing C. Incidence and diagnosis of Acute kidney injury in hospitalized adult patients: a retrospective observational study in a tertiary teaching Hospital in Southeast China. 2017. https://doi.org/10.1186/s12882-017-06226

13. Miller SA. PICO worksheet and search strategy. US National Center for Dental Hygiene Research. 2001.

14. Herrera-Méndez J, Sánchez-Velázquez LD, González-Chávez A \& Rodríguez-Terán G. Incidence of the acute renal failure in the intensive care unit at the General Hospital of Mexico: Risk factors and associated morbidity and mortality. Revista Médica Del Hospital General De México. 2015; 78(2), 62-66. https://doi.org/10.1016/j.hgmx.2015.04.0 05

15. Hulse C \& Davies A. Acute kidney injury: Prevention and diagnosis. Pediatric Kidney Disease: Second Edition. 2015; 3(2), 12231250. https://doi.org/10.1007/978-3-66252972-046

16. Pan HC, Chien YS, Jenq CC, Tsai MH, Fan $\mathrm{PC}$, Chang $\mathrm{CH}$, Chen, YC. Acute kidney injury classification for critically ill cirrhotic patients: A comparison of the KDIGO, AKIN, and RIFLE classifications. Scientific Report. 2016; 1-10. https://doi.org/10.1038/srep23022

17. Allen JC, Gardner DS, Skinner H, Harvey D, Sharman A \& Devonald MAJ. Definition of hourly urine output influences reported incidence and staging of acute kidney injury. BMC Nephrology. 2020; 21(1), 1-9. https://doi.org/10.1186/s12882-019-16782

18. Jin K, Murugan R, Sileanu FE, Foldes E, Priyanka P, Clermont G \& Kellum JA. Intensive Monitoring of Urine Output Is Associated With Increased Detection of Acute Kidney Injury and Improved Outcomes. Chest. 2017; 152(5), 972-979. https://doi.org/10.1016/j.chest.2017.05.01 1

19. Torregrosa I, Montoliu C, Urios A, AndrésCosta MJ, Giménez-Garzó C, Juan I, Miguel A. Urinary KIM-1, NGAL and L-FABP for the diagnosis of AKI in patients with acute coronary syndrome or heart failure undergoing coronary angiography. Heart and Vessels. 2015; 30(6), 703-711. https://doi.org/10.1007/s00380-014-0538z

20. Jo SK, Yang J, Hwang SM, Lee MS \& Park $\mathrm{SH}$. Role of biomarkers as predictors of 
acute kidney injury and mortality in decompensated cirrhosis. Scientific Reports. 2019; 1-9. https://doi.org/10.1038/s41598-01951053-8

21. Lima C, De Paiva Haddad LB, De Melo PDV, Malbouisson LM, Do Carmo LPF, D'Albuquerque LAC \& MacEdo E. Early detection of acute kidney injury in the perioperative period of liver transplant with neutrophil gelatinase-associated lipocalin. BMC Nephrology. 2019; 20(1), 112. https://doi.org/10.1186/s12882-0191566-9

22. Md Ralib A, Mat Nor MB \& Pickering JW. Plasma Neutrophil Gelatinase-Associated Lipocalin diagnosed acute kidney injury in patients with systemic inflammatory disease and sepsis. Nephrology. 2017; 22(5), 412-419. https://doi.org/10.1111/nep.12796

23. Parikh CR, Coca SG, Thiessen-Philbrook H, Shlipak MG, Koyner JL, Wang Z, Garg AX. Postoperative biomarkers predict acute kidney injury and poor outcomes after adult cardiac surgery. Journal of the American Society of Nephrology. 2011; 22(9),

1748-1757. https://doi.org/10.1681/ASN.2010121302

24. Schley G, Köberle C, Manuilova E, Rutz S, Forster C, Weyand M, Kientsch-engel R. Comparison of Plasma and Urine Biomarker Performance in Acute Kidney Injury. PLoS ONE. 2015; 1-24. https://doi.org/10.1371/journal.pone.014 5042

25. Uettwiller-Geiger DL, Vijayendran R, Kellum JA \& Fitzgerald RL. Analytical characteristics of a biomarker-based risk assessment test for acute kidney injury (AKI). Clinica Chimica Acta. 2016; 455, $93-$ 98.

https://doi.org/10.1016/j.cca.2016.01.012

26. Bouchard J, Malhotra R, Shah S, Kao YT, Vaida F, Gupta A, Mehta RL. Levels of protein $\mathrm{C}$ and soluble thrombomodulin in critically ill patients with acute kidney injury: A multicenter prospective observational study. PLoS ONE. 2015; 10(3), 1-14. https://doi.org/10.1371/journal.pone.01.2 0770

27. Katayama S, Nunomiya S, Koyama K, Wada M, Koinuma T, Goto Y, Shima J. Markers of acute kidney injury in patients with sepsis: The role of soluble thrombomodulin. Critical Care.2017; 21(1), 1-10. https://doi.org/10.1186/s13054-017$1815-x$

28. Murphy F \& Byrne G. The role of the nurse in the management of acute kidney injury. British journal of nursing. 2010;19, 146-52. https://doi.org/10.12968/bjon.2010.19.34 6534

29. Harty J. Prevention and management of acute kidney injury. Ulster Medical Journal. 2014; 83(3), 149-157. Retrieved from www.ums.ac.uk

30. Mohsenin V. Practical approach to detection and management of acute kidney injury in critically ill patient. Journal of Intensive Care. 2017; Vol. 5. https://doi.org/10.1186/s40560-017-0251$\mathrm{y}$

31. Gunnerson KJ, Shaw AD, Chawla LS, Bihorac A, Al-Khafaji A, Kashani K, Kellum, JA. TIMP2•IGFBP7 biomarker panel accurately predicts acute kidney injury in high-risk surgical patients. Journal of Trauma and Acute Care Surgery. 2016; 80(2), 243-249. https://doi.org/10.1097/000000000000091

32. Jamaludin TSS, Kechik NA, Saidi S \& Chan CM. Usage of Internet for Academic Purposes on University Students' Achievement: A Literature Review. Nursing \& Healthcare International Journal. 2018; 2 (1). 1-8.

33. Hamid S, Adnan W, Naing N \& Adnan A. Acute kidney injury in intensive care unit, hospital Universiti Sains Malaysia: A descriptive study. Saudi Journal Of Kidney Diseases And Transplantation. 2018; 29(5), 1109-1114. https://doi.org/10.4103/13192442243961

34. Labrador Gómez PJ, González Sanchidrián S, Labrador Gómez J, Gómez-Martino Arroyo JR, Jiménez Herrero MC, Polanco Candelario SJA, Ronco C. The role of an electronic alert system to detect acute kidney injury in hospitalized patients: DETECT-H Project. Nefrología. 2019;39(4), 379-387.

https://doi.org/10.1016/j.nefro.2018.0801 1

35. Jamaludin TSS, Nurumal MS, Saidi S, Che Hasan MK, Mohd Said NF, Samsudin NH, et al. A Narrative Review on Modern Wound Dressing for Diabetes Mellitus Wound. International Journal of Care 
Scholars [Internet]. 2020;3(2):50-6. Available from: https://journals.iium.edu.my/ijcs/index. $\mathrm{php} /$ ijcs/article/view/142/63

36. Nurumal MS, Jamaludin TSS, Mohammad NM, Che Hasan MK, Win KK. A Review on Knowledge of Diabetes and Practice of Medication Adherence Among People Living With Diabetes Mellitus. International Journal of Care Scholars [Internet]. 2020;3(1):45-54. Available from: https://journals.iium.edu.my/ijcs/index. php/ijcs/article/view/132/55 